\title{
Development of Singularities in Riemann Invariants
}

\author{
Barbara Lee Keyfitz* \\ Mathematics Department \\ University of Houston \\ Houston, Texas 77204-3476
}

May 22, 1992

\begin{abstract}
Shocks form in finite time in systems of quasilinear hyperbolic equations in one space variable which are genuinely nonlinear. We write down a simple geometric construction for systems of two equations, and use it to obtain a priori estimates for the growth of the derivatives. We also find realistic bounds on the maximum and minimum time of existence of smooth solutions for large amplitude waves in a model system of an unusual type.
\end{abstract}

\section{Contents}

1 Introduction $\quad 2$

2 The Construction 3

*Research supported by the Texas Advanced Research Program under Grant 003652124ARP and the Department of Energy, grant DE-FG05-91ER25102, and NSF, grant DMS-91-03560.

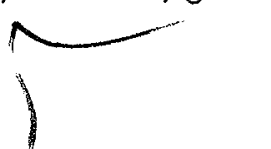


3 Applications 6

3.1 Uniform Bounds . . . . . . . . . . . . . . . 6

3.2 Singular Shock Estimates. . . . . . . . . . . . . . 8

\section{Conclusions}

\section{Introduction}

A simple construction illustrating how shocks form from smooth data of compact support, or from perturbations of Riemann data, is given for the wave equation in [7]. The geometric approach is credited to Keller and Ting, whose paper, [2], introduces the idea of integrating along characteristics to produce a problem equivalent to the scalar Burgers equation. When characteristic coordinates (global Riemann invariants) exist, this method provides both upper and lower estimates for the time of existence of smooth solutions. The construction for the nonlinear wave equation is given in detail in [7], and then generalized to wave equations with linear degeneracies. Another case, involving nonstrictly hyperbolic systems with a special structure, was studied in [3]. For equations without Riemann invariants, an alternate strategy, (see Fritz John, [1]), is to reduce the problem to blowup in a scalar ordinary differential equation, $y^{\prime}=y^{2}$.

In this note, we write down the geometric construction for any strictly hyperbolic system of two equations. The formulas we obtain differ from Keller and Ting [2, page 417, (10.9) and (10.10)], possibly because we have made the dependence on paths of integration more explicit. This generalization from the wave equation case is implicit in Klainerman and Majda's paper, [7]. Our new results are two applications: first, explicit $\mathcal{C}^{1}$ estimates on the solution, which are generalized to the case of complex characteristics in [6]. Second, we use the construction to study large-amplitude waves in a system of singular type, studied in [4], [5] and [8]. 


\section{DISCLAIMER}

This report was prepared as an account of work sponsored by an agency of the United States Government. Neither the United States Government nor any agency thereof, nor any of their employees, make any warranty, express or implied, or assumes any legal liability or responsibility for the accuracy, completeness, or usefulness of any information, apparatus, product, or process disclosed, or represents that its use would not infringe privately owned rights. Reference herein to any specific commercial product, process, or service by trade name, trademark, manufacturer, or otherwise does not necessarily constitute or imply its endorsement, recommendation, or favoring by the United States Government or any agency thereof. The views and opinions of authors expressed herein do not necessarily state or reflect those of the United States Government or any agency thereof. 


\section{DISCLAIMER}

Portions of this document may be illegible in electronic image products. Images are produced from the best available original document. 


\section{The Construction}

In Riemann invariant (characteristic) coordinates, the system is

$$
\begin{aligned}
& z_{t}+\lambda(z, w) z_{x}=0 \\
& w_{t}+\rho(z, w) w_{x}=0
\end{aligned}
$$

where $\lambda(z, w)<\rho(z, w)$ and both are smooth functions. We consider a Cauchy problem with data

$$
\begin{aligned}
& z(x, 0)=z_{0}(x) \\
& w(x, 0)=w_{0}(x)
\end{aligned}
$$

We assume sufficient smoothress of $z_{0}$ and $w_{0}$, and work in the domain of determinacy, $D(I)$, of a bounded interval, $I$, of the $x$-axis. Assuming that classical solutions exist, so that $D(I)$ is well-defined, we write down the equations for the characteristics, $X_{i}(\alpha, t)$, where $X_{1}$ is the $\lambda$ (slow) characteristic curve, in $x t$-space, originating at $(\alpha, 0)$, and $X_{2}$ the fast characteristic. Since $z$ is constant on each $X_{1}$ curve, and $w$ on each $X_{2}$ curve, by equation (1), $X_{1}$ and $X_{2}$ satisfy the parameterized ordinary differential equations

$$
\begin{aligned}
\frac{d X_{1}}{d t} & =\lambda\left(z_{0}(\alpha), w\left(X_{1}(\alpha, t), t\right)\right) \\
X_{1}(\alpha, 0) & =\alpha
\end{aligned}
$$

and

$$
\begin{aligned}
\frac{d X_{2}}{d t} & =\rho\left(z\left(X_{2}(\beta, t), t\right), w_{0}(\beta)\right) \\
X_{2}(\beta, 0) & =\beta
\end{aligned}
$$

respectively. The idea is as follows. For all $t$ such that the mappings

$$
\begin{aligned}
& \alpha \mapsto X_{1}(\alpha, t)=x \\
& \beta \mapsto X_{2}(\beta, t)=x
\end{aligned}
$$

remain invertible, solutions $z$ and $w$ satisfy

$$
(z(x, t), w(x, t))=(z(\alpha, \beta), w(\alpha, \beta))=\left(z_{0}(\alpha), w_{0}(\beta)\right)
$$


in $D(I)$. Furthermore, the mappings (5) reduce to the identity at $t=0$. One derives an equation for $\partial X_{1} / \partial \alpha$ (and, similarly, for $\partial X_{2} / \partial \beta$ ) by differentiating (3) (or (4)) with respect to $\alpha$ (or $\beta$ ):

$$
\frac{d}{d t}\left(\frac{\partial X_{1}}{\partial \alpha}\right)=\frac{\partial \lambda}{\partial z}\left(z_{0}(\alpha), w\left(X_{1}(\alpha, t), t\right)\right) z_{0}^{\prime}(\alpha)+\frac{\partial \lambda}{\partial w} \frac{\partial w}{\partial x} \frac{\partial X_{1}}{\partial \alpha}
$$

The second idea in this construction is that the term $\partial w / \partial x$ in (6) can be replaced by the derivative of $w$ along the curve $X_{1}$. From the second equation in (1), the two partial derivatives of $w$, and hence all other directional derivatives, are functionally related. This results in

$$
\left.\frac{d w}{d t}\right|_{X_{1}}=\frac{\partial w}{\partial t}+\lambda \frac{\partial w}{\partial x}=-(\rho-\lambda) \frac{\partial w}{\partial x} .
$$

The coefficient of $\partial X_{1} / \partial \alpha$ in (6) can thus be rewritten:

$$
\frac{\partial \lambda}{\partial w} \frac{\partial w}{\partial x}=-\frac{1}{\rho-\lambda} \frac{\partial \lambda}{\partial w} \frac{d}{d t} w\left(X_{1}(\alpha, t), t\right)=-\frac{d F}{d t}\left(z_{0}(\alpha), w\left(X_{1}(\alpha, t), t\right)\right)
$$

where differentiation is along $X_{1}$, and $F$ is a function of $z$ and $w$ obtained by partial integration of

$$
\frac{\partial F}{\partial w}=\frac{1}{\rho-\lambda} \frac{\partial \lambda}{\partial w}
$$

with respect to $w$. In special cases (nonlinear wave equations, for example) the primitive, $F$, can be evaluated explicitly.

Abbreviating $\partial X_{1} / \partial \alpha$ in (6) by $y(t)$, and the first term on the right in (6) by $q$, we get a linear ordinary differential equation:

$$
\frac{d y}{d t}+F^{\prime}(t) y(t)=q(t)
$$

Here, and below, $F(t)=F\left(z_{0}(\alpha), w\left(X_{1}(\alpha, t), t\right)\right)$. We solve (8) by means of the integrating factor $e^{F(t)}$; since $y(0)=1$, we obtain the basic formula

$$
y(t) \equiv \frac{\partial X_{1}}{\partial \alpha}=e^{F(0)-F(t)}\left\{1+\int_{0}^{t} e^{F(s)-F(0)} q(s) d s\right\} .
$$

The expression in braces in (9) is

$$
1+z_{0}^{\prime}(\alpha) e^{-F(0)} Q(t)
$$


where

$$
Q(t)=\int_{0}^{t} \frac{\partial \lambda}{\partial z}\left(z_{0}(\alpha), w\left(X_{1}(\alpha, s), s\right)\right) e^{F(s)} d s .
$$

In one application, we are interested in singularities in the mapping (5). Now, a singularity arises if $y(t)<0$ at some $(\alpha, t)$, while the solution is smooth as long as both $y(t)$ and its counterpart $\partial X_{2} / \partial \beta$ are positive. To estimate these times, suppose $z_{0}$ and $w_{0}$ are bounded, defining a rectangle $R$ in the $z w$ plane:

$$
\left.\begin{array}{l}
z_{\min } \leq z_{0} \leq z_{\max } \\
w_{\min } \leq w_{0} \leq w_{\max }
\end{array}\right\} R
$$

on $I$; these bounds persist in $D(I)$ as long as the solution is smooth. Furthermore, if we assume the system is genuinely nonlinear, then $\partial \lambda / \partial z \neq 0$ also, and this term is bounded on $R$; replacing $z$ by $-z$ if appropriate, we have

$$
0<\ell_{1} \leq \frac{\partial \lambda}{\partial z} \leq \ell_{2}
$$

on $R$. Similarly $F$ will be bounded above and below on $R$, and $e^{F}$ is positive:

$$
0<f_{1} \leq e^{F(z, w)} \leq f_{2}
$$

Hence $0<\ell_{1} f_{1} \leq Q^{\prime}(t) \leq \ell_{2} f_{2}$ on $R$ and

$$
0<\ell_{1} f_{1} t \leq Q(t) \leq \ell_{2} f_{2} t
$$

Now $(10)$ is positive along $X_{1}(\alpha, t)$ for all $t>0$ if $z_{0}^{\prime}(\alpha)>0$. For $\alpha$ such that $z_{0}^{\prime}(\alpha)<0,(10)$ is positive if

$$
Q(t)<\frac{e^{F\left(z_{0}(\alpha), w_{0}(\alpha)\right)}}{-z_{0}^{\prime}(\alpha)}
$$

and thus remains positive as long as

$$
t \leq \frac{e^{F\left(z_{0}(\alpha), w_{0}(\alpha)\right)}}{-z_{0}^{\prime}(\alpha) \ell_{2} f_{2}}
$$

However, a singularity arises when (10) becomes negative; that is, by the same calculation, when $z_{0}^{\prime}(\alpha)<0$ and

$$
t \geq \frac{e^{F\left(z_{0}(\alpha), w_{0}(\alpha)\right)}}{-z_{0}^{\prime}(\alpha) \ell_{1} f_{1}}
$$


Reversing the roles of $z$ and $w$ and of the $X_{1}$ and $X_{2}$ characteristics, one obtains bounds for the time interval on which the other mapping, $\beta \mapsto X_{2}$, will be invertible. Define $G$ by

$$
\frac{\partial G}{\partial z}=-\frac{1}{\rho-\lambda} \frac{\partial \rho}{\partial z}
$$

and suppose $e^{G}$ is bounded below and above by $g_{1}$ and $g_{2}$ on $R$; again choose $w$ so that $\partial \rho / \partial w$ is positive; let it be bounded by $r_{1}$ and $r_{2}$ on $R$. Then we obtain a minimum time of existence

$$
t \leq \frac{e^{G\left(z_{0}(\beta), w_{0}(\beta)\right)}}{-w_{0}^{\prime}(\beta) r_{2} g_{2}} .
$$

and an upper bound that smooth solutions cannot exist for

$$
t \geq \frac{e^{G\left(z_{0}(\beta), w_{0}(\beta)\right)}}{-w_{0}^{\prime}(\beta) r_{1} g_{1}} .
$$

In this case, again, we need look only at $X_{2}$ characteristics where $w_{0}^{\prime}<0$. The analog of $(10)$ is

$$
1+w_{0}^{\prime}(\beta) e^{-G(0)} Q_{1}(t)>0
$$

where

$$
Q_{1}(t)=\int_{0}^{t} \frac{\partial \rho}{\partial w}\left(z\left(X_{2}(\beta, s), s\right), w_{0}(\beta)\right) e^{G(s)} d s .
$$

\section{Applications}

\subsection{Uniform Bounds}

If we consider data which is close to a constant in the $\mathcal{C}^{1}$ norm:

$$
\sup _{I}\left(\left|z_{0}(x)-\bar{z}\right|,\left|w_{0}(x)-\bar{w}\right|,\left|z_{0}^{\prime}(x)\right|,\left|w_{0}^{\prime}(x)\right|\right) \leq \epsilon,
$$

then solutions do not develop singularities within a time of order $1 / \epsilon$, provided the system is strictly hyperbolic in the closed rectangle $R_{\epsilon}$ so defined. The terms used to estimate $Q(t)$ and $Q_{1}(t)$ depend only on the $\mathcal{C}^{0}$ norm of $z_{0}$ and $w_{0}$ and have order one; derivatives of $z_{0}$ and $w_{0}$ influence the bounds 
only where they appear explicitly in (10) and (16). In particular, one can produce order one bounds on the time in several ways, by controlling the size and oscillation of the initial data and their derivatives.

We obtain a priori bounds on the $\mathcal{C}^{1}$ norm of solutions to (1) as follows.

Proposition 1 Let $z(x, t)$ and $w(x, t)$ be $\mathcal{C}^{1}$ solutions of $(1)$ in $D(I)$, with data satisfying (17). Then, provided $\epsilon$ is sufficiently small and given any $\kappa>1$, there is a time $\tau$, depending only on $\epsilon, \kappa$ and geometric constants, such that

$$
\sup _{I(r)}\left(|z(x, t)-\bar{z}|,|w(x, t)-\bar{w}|,\left|\partial_{x} z(x, t)\right|,\left|\partial_{x} w(x, t)\right|\right) \leq \kappa \epsilon,
$$

where $I(\tau)$ is the intersection of $D(I)$ with the strip $0 \leq t \leq \tau$.

Proof: The first two quantities in (18) are bounded in $D(I)$ for any $\kappa \geq 1$, as we have noted. For the third, we have

$$
\frac{\partial z}{\partial x}=\frac{\partial z}{\partial \alpha} \frac{\partial \alpha}{\partial x}=\frac{z_{0}^{\prime}(\alpha)}{y(t)}
$$

where $y$ is evaluated on $X_{1}(\alpha, \cdot)$. Hence $\left|z_{x}\right| \leq \kappa \epsilon$ where

$$
\kappa=\sup \left(\frac{1}{y}\right) .
$$

Let $\ell=\sup _{R_{e}}\left|\lambda_{z}\right| ;$ then, from (9) and (10),

$$
\frac{1}{y(t)} \leq f\left(1-\epsilon t\left(\frac{\ell f_{2}}{f_{1}}\right)\right)^{-1}=f(1-K \epsilon t)^{-1},
$$

say, and $f \equiv \sup \left(e^{F(t)-F(0)}\right)$ can be estimated from

$$
|F(t)-F(0)|=\left|\int_{0}^{t} F^{\prime}(s) d s\right| \leq L t,
$$

on $D(I)$. Here

$$
L \equiv \sup _{D(I)}\left|F^{\prime}(t)\right|=\sup _{R_{\epsilon}}\left|\frac{\lambda_{w}}{\rho-\lambda}\right|
$$


So

$$
\left|z_{x}\right| \leq \epsilon(1-K \epsilon t)^{-1}(1+L t+\cdots) \leq \epsilon(1+M t)
$$

where, for sufficiently small $\epsilon, M=L+K \epsilon+\mathcal{O}\left(\left(L^{2}+\epsilon^{2}\right) t\right)$ depends on $L$, $\inf _{R_{\epsilon}}(\rho-\lambda)$, and the length of $I$. Hence for any $\kappa>1$, the third bound in (18) holds with $\tau=(\kappa-1) / M$. We get a similar estimate for $\left|w_{x}\right|$. From (1), we also find bounds on $\left|z_{t}\right|$ and $\left|w_{t}\right|$ on $I(\tau)$ :

$$
\sup \left(\left|z_{t}\right|,\left|w_{t}\right|\right) \leq \Lambda \kappa \epsilon \text {. }
$$

where $\Lambda \equiv \sup _{R_{e}}(|\lambda|,|\rho|)$.

This proves Proposition 1.

\subsection{Singular Shock Estimates}

Finally, we apply the construction to a model system which exhibits singular shocks. The system, studied in several papers of Keyfitz and Kranzer ([4], [5], [8]), has the feature that, although it is genuinely nonlinear and strictly hyperbolic, the wave speeds are not separated. We have studied extensively a prototype equation where the speeds differ by a constant. In Riemann invariant coordinates the system is

$$
\begin{aligned}
& \frac{\partial z}{\partial t}+(u-1) \frac{\partial z}{\partial x}=0 \\
& \frac{\partial w}{\partial t}+(u+1) \frac{\partial w}{\partial x}=0 .
\end{aligned}
$$

Here global Riemann invariants are

$$
z=v-\frac{1}{2} u^{2}+u, \quad w=-v+\frac{1}{2} u^{2}+u,
$$

with inverse mapping

$$
u=\frac{z+w}{2}, \quad v=\frac{z-w}{2}+\left(\frac{z+w}{8}\right)^{2} .
$$

The characteristic speeds are $(z+w) / 2 \mp 1 ;$ now, $\rho-\lambda=2$, and $\partial F / \partial w=$ $\frac{1}{2} \partial \lambda / \partial w=\frac{1}{4}$. Let

$$
F(z, w)=\frac{w}{4} \quad \text { and } \quad G(z, w)=-\frac{z}{4} .
$$


We consider large amplitude data as part of a program to study singular shocks. For example, in [5] we consider formation of shocks from smooth initial data connecting two states

$$
U_{0}=\left(u_{0}, v_{0}\right) \text { and } U_{1}=\left(u_{0}+\frac{a}{\alpha_{1} \sqrt{\epsilon}}, v_{0}+\frac{a^{2}}{\alpha_{1}^{2} \epsilon}\right) .
$$

The estimates (12), (13), (14), and (15) of the last section are not useful because they give upper and lower bounds which are very far apart. However, a more careful analysis is possible. We consider a single case here, and make some simplifying assumptions, to illustrate this analysis.

Let $U_{0}=0$ and absorb $\alpha_{1}$ into $a$. The important distinction is between $a>0$ and $a<0$. Define

$$
\begin{aligned}
& z\left(U_{0}\right)=0, \quad z\left(U_{1}\right)=\frac{a^{2}}{2 \epsilon}+\frac{a}{\sqrt{\epsilon}} \equiv Z_{1}, \\
& w\left(U_{0}\right)=0, w\left(U_{1}\right)=-\frac{a^{2}}{2 \epsilon}+\frac{a}{\sqrt{\epsilon}} \equiv W_{1} ;
\end{aligned}
$$

and set

$$
z_{0}(x)=Z_{1} x, \quad w_{0}(x)=W_{1} x
$$

on $0 \leq x \leq 1$. We prove the following.

Proposition 2 Consider equation (19) with initial data $z_{0}$, wo joining the state $z=w=0$ on the left to the state $\left(Z_{1}, W_{1}\right)$ on the right by a continuous straight-line interpolation. Then there are constants $K_{1}$ and $K_{2}$, independent of $\epsilon$, such that if $a>0$ the solution remains continuous, with bounded derivatives, until time $t=K_{1} / \sqrt{\epsilon}$. If $a<0$ there is no continuous solution for $t \geq K_{2} \sqrt{\epsilon}$.

Proof: We ignore the unimportant modifications which must be made at jump discontinuities in the derivatives of $z_{0}$ and $w_{0}$. Note that $z_{0}$ is increasing and $w_{0}$ decreasing, for $a$ of either sign. The last assertion of the proposition follows immediately by noting that the characteristics originating on the intervals where the data are constant have speeds \pm 1 , on the left, and $(a / \sqrt{\epsilon}) \pm 1$, on the right. If $a<0$ and $\epsilon$ is small enough the extreme characteristics intersect within the stated time. 
Now consider $a>0$. From the arguments in Section 2, singularities will not develop in $z$, and will not develop in $w$ until a time $t$ such that

$$
1+\frac{1}{2} w_{0}^{\prime}(\beta) e^{\frac{1}{4} z_{0}(\beta)} \int_{0}^{t} e^{-\frac{1}{4} z\left(X_{2}(\beta, s), s\right)} d s=0 .
$$

Now, we may write $z\left(X_{2}(\beta, s), s\right)=z_{0}(A(\beta, s))$, where $A(\beta, t)$ is defined by

$$
X_{1}(A, t)=X_{2}(\beta, t)
$$

note that $A>\beta$. Then the integral in (20) can be written

$$
Q_{1}(t)=\int_{0}^{t} e^{-\frac{1}{4} z_{0}(A(\beta, s))} d s=\int_{\beta}^{\beta_{1}}\left(\frac{d s}{d A}\right) e^{-\frac{1}{4} z_{0}(A)} d A
$$

where the upper limit of integration is between $\beta$ and $x=1$. Because $z_{0}$ is linear, the integrand of $Q_{1}$ contains a rapidly decreasing exponential. We recall the formula (easily confirmed)

$$
\int_{\beta}^{\beta_{1}} f(A) e^{-K A} d A=e^{-K \beta}\left[\frac{f(\beta)}{K}+\frac{E}{K^{2}}\right] .
$$

and note that at $A=\beta, f(\beta)=d s / d A(\beta)=\frac{1}{2}$ from (21). Here $K=\frac{1}{4} Z_{1}$, and $E$ is an error term which depends on $t$ (through $\beta_{1}$ ) but is bounded in $\epsilon$. Thus $Q_{1}$ becomes

$$
Q_{1}(t)=\frac{e^{-\frac{1}{4} Z_{1} \beta}}{\frac{1}{4} Z_{1}}\left(\frac{1}{2}+\frac{E}{\frac{1}{4} Z_{1}}\right)
$$

note that to a first approximation $Q_{1}$ is independent of $t$.

When we evaluate (20) to highest order we get

$$
1+\frac{1}{2} W_{1} e^{\frac{1}{4} Z_{1} \beta} \frac{1}{2} \frac{e^{-\frac{1}{4} Z_{1} \beta}}{\frac{1}{4} Z_{1}}=1+\frac{W_{1}}{Z_{1}}=\frac{Z_{1}+W_{1}}{Z_{1}}=\frac{2 a}{Z_{1} \sqrt{\epsilon}}
$$

Since $a>0$ this quantity is strictly positive (albeit of order $\sqrt{\epsilon}$ ), and the solution is smooth. Including the error term and noting that $E$ grows at most linearly with $t$ gives the required estimate when $a>0$.

This completes the proof. 
Studying piecewise constant box approximations to singular shocks in [5], we examined two types of discontinuities: those connecting asymptotically infinite states with finite states, as analysed here, and those connecting a large-amplitude state on the left (with $u>0$ ) to another on the right (with $u<0$ ). Proposition 2 shows that the first type of discontinuity is not compressive if $a>0$. The second type of connection, which is compressive, leads to formation of singular shocks; but if the left and right states are reversed (that is, $a>0$ is replaced by $a<0$ ), the argument of Proposition 2 can be adapted to show that shocks do not form.

\section{Conclusions}

We have rederived a formula and used it to estimate the time it takes for shocks to develop from smooth data in a system of two conservation laws. This formula gives useful $\mathcal{C}^{1}$ bounds on solutions. We also adapted the formula to examine large-amplitude waves for problems involving singular shocks [5], in a context where estimates for small-amplitude waves are not sharp enough.

An interesting difference between the problems analysed in [2] and ours is that our formula contains exponential terms (resulting from the integrating factors $F$ and $G$ ), while the corresponding wave equation formulas do not (even when corrected, [7]). This happens because in typical nonlinear wave equations $F$ and $G$ are logarithmic, and their exponentials are of order one in the variables. Our singular shock problem has the feature, very different from the nonlinear wave equation, that the difference $\rho-\lambda$ between wave speeds is constant; $F$ and $G$ are of the order of the speeds themselves, and their exponentials exhibit exponential growth in the wave amplitudes.

The formula originally found by Keller and Ting was rederived by Klainerman and Majda for the wave equation, and in that paper, [7], was then used to study a different problem, namely the formation of shocks in systems which are not genuinely nonlinear. That pathology is different, as far as we know, from the genuinely nonlinear problem we study here. We have suggested the name 'same variation' to describe nonlinear systems where the wave speeds are of this type, and where formation of singular shocks is to be expected. 


\section{References}

[1] F. John, 'Formation of singularities in one-dimensional nonlinear wave propagation', Comm. Pure Appl. Math., XXVII, 1974, 377-405.

[2] J. B. Keller and L. Ting, 'Periodic vibrations of systems governed by nonlinear partial differential equations', Comm. Pure Appl. Math., XIX, $1966,371-420$.

[3] B. L. Keyfitz and H. C. Kranzer, 'Non-strictly hyperbolic systems of conservation laws: formation of singularities', in Nonlinear Partial Differential Equations, (ed. J. A. Smoller), Contemporary Mathematics, 17, American Mathematical Society, Providence, 1983.

[4] B. L. Keyfitz and H. C. Kranzer, 'A viscous approximation to a system of conservation laws with no classical Riemann solution', in Nonlinear Hyperbolic Problems (C. Carasso, ed), Springer, LNM 1402, 1989, 185197.

[5] B. L. Keyfitz and H. C. Kranzer, 'Spaces of weighted measures for conservation laws with singular shock solutions', in preparation, 1992.

[6] B. L. Keyfitz and M. Lopes, 'A geometric study of shocks in equations that change type', in preparation, 1992.

[7] S. Klainerman and A. J. Majda, 'Formation of singularities for wave equations including the nonlinear vibrating string', Comm. Pure Appl. Math., XXXIII, 1980, 241-263.

[8] H. C. Kranzer and B. L. Keyfitz, 'A strictly hyperbolic system of conservation laws admitting singular shocks', in Nonlinear Evolution Equations that Change Type, (ed. Keyfitz and Shearer), IMA Vol 27, Springer, $1990,107-125$. 